\title{
An Overview of Change Management within Manufacturing Environment
}

\author{
SYED M Hasan ${ }^{1, a}$ and SATYA Shah ${ }^{2, b}$ \\ ${ }^{1}$ Centre of Innovative Product Development and Manufacturing \\ Faculty of Engineering and Science, University of Greenwich \\ Medway Campus, Chatham, Kent - ME4 4TB, United Kingdom \\ ${ }^{2}$ Centre of Innovative Product Development and Manufacturing \\ Faculty of Engineering and Science, University of Greenwich \\ Medway Campus, Chatham, Kent - ME4 4TB, United Kingdom \\ as.m.hasan@gre.ac.uk, ${ }^{\mathrm{b}}$ s.shah@gre.ac.uk
}

Keywords: Change Management, New Product Development, Supply Chain, Business Process Modeling, Business Process Simulation, Integration

\begin{abstract}
In this competitive world, where features like time to market, new technology and change management are the features that can affect the performance of New Product Development (NPD). Companies tend to use a conventional approach to NPD by assigning representatives from support functions to review and recommend changes as projects evolve. This approach has, in recent years, been questioned since it is a costly and time-consuming approach due to its iterative nature. It is argued that the change management process can reduced the negative effect and has the ability to support the functions of a supply chain to a greater extent and also earlier in the NPD process. This paper focuses on different change management techniques to support the required changes by management to integrate the NPD within supply chain (SC).
\end{abstract}

\section{Introduction}

It is well recognized that the NPD process not only enables management to coordinate the flow of new products efficiently, but also to assist in the ramp-up of sourcing, manufacturing, distribution, and other sales-related activities that support the commercialization of the product. Therefore, the traditional NPD functions must be coordinated with the support functions [1]. For this change aspect, new practices have emerged in the area of change management (CM) to address this lead-time issue. These practices include simulation and concurrent design. Simulation involves a multifunctional development team. This development team is highly structured and infused with greater responsibility and authority. However, concurrent design has mostly focused on internal collaboration while today's global competition may require for concurrent design to be a collaboration in the entire demand-supply chain, as it is an important key to success and profitability. The terms "design for manufacturing" and "design for supply chain" are quite often used to imply that the traditional NPD functions are aligned and coordinated with other main functions in the company and in the extended enterprise.

\section{Literature Review}

Although, companies are focussing on any type of change, they have to go through the aspect of change management (CM) within their respective environments. An important focus of $\mathrm{CM}$ has been on overcoming resistance to change. For example, [2] define CM as "the leadership and direction of the process of organisational transformation - especially with regard to human aspects of overcoming resistance to change". It is interesting to note that although change is an implicit aspect of business improvement, references to CM publications within the operations and supply chain improvement literature are scarce. Analysed from a different perspective, [3] comments on the tendency for 
academic CM literature to avoid the terminology of management tools and techniques. He argues that there needs to be a far greater acknowledgment of the inter connections between tools and techniques and CM models, theories and concepts.

Existing Model. One CM model that makes this connection more explicit is [4] TROPICS test. This specific model is developed to feel of change, including the choice of solution methodology, where "hard" defines system-based, mechanistic solution methodology, and "soft" defines an organisational development, complex solution methodology.

Table 1: TROPICS methodology [4]

\begin{tabular}{|l|l|l|}
\hline \multicolumn{1}{|c|}{ Tropics Factor } & "Hard" Solution Methodology & "Soft"Solution Methodology \\
\hline Timescales & $\begin{array}{l}\text { Clearly defined: Short to medium } \\
\text { term }\end{array}$ & Ill define: Medium to long term \\
\hline Resources & $\begin{array}{l}\text { Clearly defined and reasonably } \\
\text { fixed }\end{array}$ & Unclear and variable \\
\hline Objectives & Objective and quantifiable & Subjective and visionary \\
\hline Perceptions & Shared by those affected & Creates conflicts of interest \\
\hline Interest & Limited and well define & Widespread and ill defined \\
\hline Control & Within the managing group & Shared out with the group \\
\hline Source & Originates internally & Originates externally \\
\hline
\end{tabular}

In terms of taking a more strategic and holistic approach to experience design, [5] divided experience design into four phases:

(1) Acquisition of service experience design skills;

(2) Data collection and analysis;

(3) Service clue design; and

(4) Implementation and verification.

Later, 2004 Carbone suggested five steps [6]:

(1) Build a diverse design team.

(2) Drill down to the experience core.

(3) Focus on clues.

(4) Develop the experience narrative or story line.

(5) Prioritise implementation opportunities.

In 2007 Berry and Carbone proposed a five-step approach [7]:

(1) Identify the emotions that evoke customer commitment.

(2) Establish an experience motive.

(3) Inventory and evaluate experience clues.

(4) Determine the experience gap.

(5) Close the experience gap \& monitor execution.

Kotter's "eight step" change model. Kotter's framework and analysis of change, based primarily on organisational change in the corporate sector, has been articulated and adapted since the mid-1990s. It is well known and widely quoted and applied. The language and philosophy of Kotter's approach appears in many iterations and variations in the literature of organisational change. Kotter's prescription for success is to recognise the importance of a staged and sequential approach, not to rush and/or to fall victim to the illusion of speed, and to look out for and correct the pitfalls that accompany each of these stages of change:

(1) Establish a sense of urgency - about the need to make changes.

(2) Form a powerful high level coalition to guide and lead the changes - a group with enough power and influence in the organisation to lead \& promote the change effort.

(3) Create a vision of the organisation's future - to help focus and direct the change. 
(4) Communicate that vision widely, repeatedly and consistently - from the leadership level down through all organisational levels, in language and in actions and behaviours.

(5) Empower people in the organisation to act on the vision - remove obstacles to change, improve processes and systems, encourage and enable people to take risks, engage in non-traditional thinking and activities.

(6) Plan for visible short-term performance improvements - enable these to occur and recognise their achievement and the work of those who have enabled that achievement.

(7) Consolidate improvements and produce more change - as change takes effect build on the credibility and confidence that results, extending the reform or structures, systems and processes and encouraging and growing change agents in the organisation.

(8) Institutionalise new approaches - clearly articulate the connections between the new ways of working and organisational successes, encourage and develop on going leadership of change and anchor the changes into the organisational culture.

Doppelt's "wheel of change" model. By contrast Doppelt's [8] analysis provides a newer and less widely known approach to understanding and approaching organisational change. This analysis of the dynamics of organisational change is drawn from wide ranging and long term analysis of organisations. It views the process of change as being a cycle or a wheel. He articulates seven points at which interventions may be made or leverage applied to effect change. Significantly, and in variance to Kotter's view of the primacy of strict ordering of sequential steps in the process of change, his argument was interventions may be made at any point in the cycle, provided that all steps are carried through.

Doppelt acknowledges change as a messy and far from linear process and suggests that, while implementation of all seven components or leverage points is essential for achieving organisational effectiveness, it is possible to enter the change cycle at any point and to work with any of the leverage points and with vary degrees of attention to each.

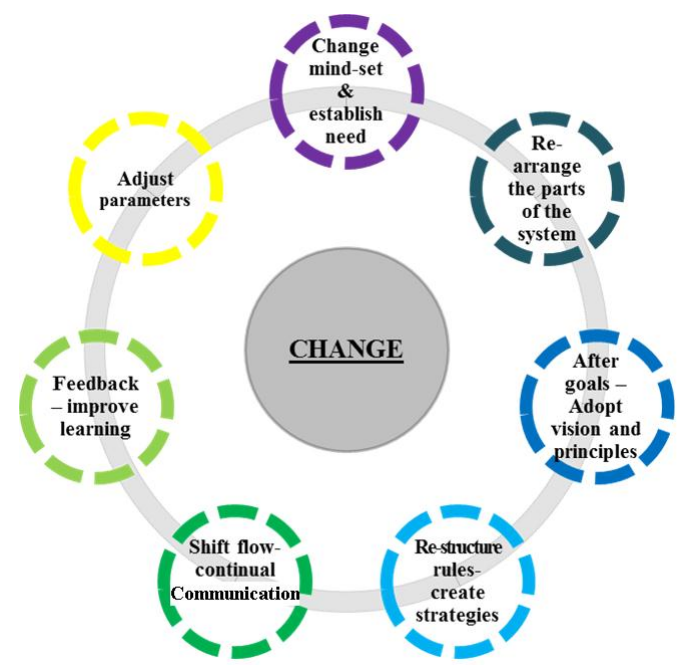

Fig. 1: Doppelt's wheel of change. [8]

Business Process Modeling. Business Process Modelling (BPM) is a structured approach to analyse and continually improve fundamental activities such as manufacturing, marketing, communications and other major elements of a company's operation. Essentially,

BPM is concerned with the main aspects of business operations where there is high leverage and a big proportion of added value. BPM has to be governed by the following rules:

- Major activities have to be properly mapped and documented.

- BPM creates a focus on customers through horizontal linkages between key activities.

- BPM relies on systems and documented procedures to ensure discipline, consistency and repeatability of quality performance.

- BPM relies on measurement activity to assess the performance of each individual process, set targets and deliver output levels which can meet corporate objectives. 
- BPM has to be based on a continuous approach of optimization through problem solving and reaping out extra benefits.

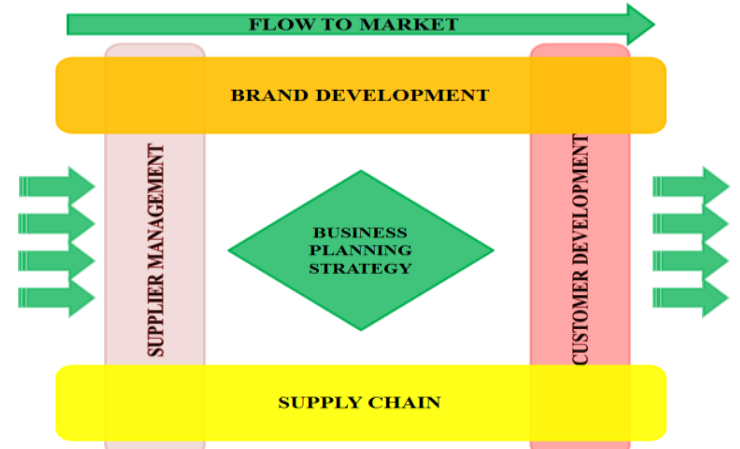

Fig. 2: Activities in Business Process Management [9]

BPM emerged as a succeeding concept to Total Quality Management (TQM) in the 1980s and Business Process Reengineering (BPR) in the 1990s. Following BPR, several IT systems such as Enterprise Resource Planning (ERP) and Customer Relationship Management (CRM) gained organizational focus [10]. Given this history of IT systems, BPM initially focused on technical, IT-related aspects of business processes and their design via technology. Despite an early awareness by [14], researchers have only in recent years more broadly considered BPM to be an integrated approach that moves beyond purely an IT focus [11].

Business Process Simulation. Business process simulation (BPS) used to be the way to do the analysis of manufacturing systems, but is now being utilised in the management of change in a different way of manufacturing and service platforms. Simulation can be referred to different types of model like spread sheet modelling, discrete event simulation and a system dynamic simulation.

BPS is used to assist decision making by providing a tool that allows the 'AS IS' behaviour of a system to be analysed. It helps to predict the performance of that system under a number of scenarios created by the decision maker. Research indicates how BPS could provide support in a process centred management approach to change [12]. The model shows the use of simulation not only to predict the performance of the "to-be" design before resources are committed, but also to use the technique to construct a model of the "as-is" state in order to understand the process and measure the variation that takes place in key performance measures.

The benefits of BPS. The main advantage of the BPS analysis is that it allowed the incorporation of variability and interdependence factors in order to obtain an accurate outline of process performance. The simulation could predict process performance along a number of measures such as lead-time, resource utilisation and cost. In this case a cost element was used to estimate expenditure by staff role, process and arrest type. Once built, the BPS allowed analysis of many potential new designs through its "what-if" capability with little extra effort. For example a number of booking-in process redesigns could be analysed in advance using the model, before implementation.

Limitations of BPS for BPR. A major barrier to many organisations in using BPS is the preparation needed in the successful introduction of the technique to the organisation. The potential that process mapping will lead to too much emphasis on operationalizing existing processes, rather than conceptualising a new design has been recognised and BPS could be said to increase the scope for over-analysis. However, it should be recognised that when estimating the amount of resource required to construct a BPS, that there should be no attempt to model every aspect of the area of study, but the level of detail and scope of the model should be judged according to the study objectives. Thus building a sophisticated model must not become the objective of the exercise, the model should be built with just enough detail to provide information on which to make decisions.

One limitation of BPS in the context of BPR projects is that the BPR team must be careful not to create a "to-be" design based solely on "tweaking" the "as-is" simulation model. "Simulation is most useful in comparing 'as-is' and 'to-be' models, and validating and ensuring the completeness of the 'to-be' process model. However, beyond this simulation has limited ability in creating a 'to-be' model" as shown through research. Thus simulation will not create a new design and design ideas 
should not be constrained by the complexity of changing the model to simulate the new design. Design ideas should drive simulation design, not the other way around.

It is also noted that simulation is most useful towards the analysis of stable business processes; however less useful for dynamic systems that do not reach equilibrium. This may point to potential difficulties in statistical analysis of service systems that are less likely to reach equilibrium than a manufacturing process.

\section{Conclusion}

The paper presents here different forms of change management techniques that are required in supporting the management decisions towards the integration of new product development (NPD) within existing supply chain (SC) environments. There is some evidence through the literature which suggests that manufacturing companies prefer to use conventional approaches towards new product development environments. However, these approaches tend to be time consuming and costly as it is iterative in nature. Whereas, in today changing global and competitive environment, features such as time to market, new technology and change management are important towards considering new product development aspects. It is also evident from the literature that many suppliers' learning is not just focused entirely on the process followed for the development, but also towards their customer knowledge and it is this integration of "new knowledge" which leads to better performance of new products within.

\section{References}

[1] P. Hilletofth, D. Ericsson, and M. Christopher, Demand chain management: a Swedish industrial case study, Industrial Management \& Data Systems, 109, No. 9 (2009), 1179-1196.

[2] R. Fincham, and P. Rhodes, Principles of Organizational Behavior, 4th ed., Oxford University Press 2005.

[3] T. Hughes, Regaining a seat at the table: marketing management and the e-service opportunity, Journal of Services Marketing, 21, No 4 (2007), 270-280.

[4] R. A. Paton and J. McCalman , Change Management: A Guide to Effective Implementation, Second ed., 2000.

[5] L. P. Carbone, S. H. Haeckel, Engineering customer experiences, Journal of Marketing Management, 3 No. 3 (1994), 8-19.

[6] L. P. Carbone, Clued In, FT Prentice Hall, New Jersey, 2004.

[7] L. L. Berry and L. P. Carbone, Build loyalty through experience management, Quality Progress, 40 No. 9 (2007), 26-32.

[8] B. Doppelt, Overcoming the seven sustainability blunders, The System thinker, 14 No.5 (2003).

[9] M. Zairi, Business process management: Boundary less approach to modern competitiveness, Journal of Business Process management, 3 No. 1 (1997) 64-80.

[10]J. Jeston and J. Nelis, Business Process Management: Practical Guidelines to Successful Implementations, Elsevier, Oxford, 2008.

[11]P. Harmon, The scope and evolution of business process management, in J. vom Brocke and M. Rosemann (Eds.), Handbook on Business Process Management: Introduction, Methods and Information Systems, Springer, Berlin, 1 (2010).

[12] R. Kalakota and M. Robinson, E-Business 2.0: Roadmap for Success, Addison-Wesley, Boston, 2002. 